\title{
ANSIEDAD ESCÉNICA E INTELIGÊNCIA EMOCIONAL EN INTÉRPRETES MUSICALES EN FUNCIÓN DEL TIPO DE EDUCACIÓN RECIBIDA: UNA COMPARATIVA ENTRE INTÉRPRETES CLÁSSICOS Y MODERNOS
}

\section{Ansiedade cênica e inteligência emocional em interpretes musicais em função do tipo de educação recebida: uma comparação entre intérpretes clássicos e modernos}

\author{
Scenicanxietyandemotionalintelligence in musicians, \\ dependingonthetypeofeducationreceived: a \\ comparisonbetweenclassicalandmoderninterpreters
}

Elvira Martínez Belmonte*

Juan Robles de la Puente**

\begin{abstract}
Resumen
El presente trabajo se centra en el estudio de la ansiedad escénica y la inteligencia emocional en músicos, realizando una comparativa entre intérpretes de música clásica e intérpretes de música moderna, observándose diferencias significativas sólo en la dimensión ansiedad estado, referida en este caso a situaciones de actuación en público.La ansiedad escénica está presente, en mayor o menor medida, en la vida de todo intérprete musical. En ocasiones, si se da en exceso puede llegar a suponer un gran problema para algunos músicos. Por ello creemos que resulta muy importante estudiarla y profundizar en su conocimiento con el objetivo de comprender mejor sus causas y ayudar a disminuir sus efectos negativos.Entender cómo se puede desarrollar y potenciar la inteligencia emocional también puede ser de mucha utilidad para los intérpretes musicales, que con frecuencia se enfrentan a situaciones en las que deben lidiar con diversos cambios emocionales y pueden llegar a verse sobrepasados por ellos. Poder entender las propias emociones y sus cambios creemos que puede ayudar a un mayor conocimiento personal y a un mejor control de las mismas, que permita que el músico pueda sentirse cómodo tocando y a la vez sea capaz de transmitir lo que desea al público.
\end{abstract}

\section{PALABRAS-CLAVE: Ansiedad. Musica. Inteligencia emocional.}

\section{Resumo}

Este artigo centra-se no estudo da ansiedade de desempenho e músicos de inteligência emocional, realizando umacomparação entre músicos clássicos e artistas da música moderna, nãoforam observadas diferenças significativas apenas no estado dimensãoansiedade, referida neste caso situaçõespúblico.Laansiedade de desempenhodesempenho está presente, emmaiorou menor grau na vida de cada performer musical. Àsvezes, se dado emexcesso pode ser visto como um grande problema para alguns músicos. Por isso, acreditamos que é muito importante para estudar e aprofundar os seusconhecimentos, a fim de melhorcompreender as suas causas e ajudar a reduzir os

\footnotetext{
*Titulada Superior de Música en la especialidad de Piano, y Máster en Interpretación Musical e Investigación Performativa. Trabaja en Piano Academy of Ireland. E-mail: elvira.marbel@ gmail.com

**Doctor en psicología y músico profesional. Universidad Alfonso X El Sabio. Facultad de Música y Artes Escénicas.Su investigación se centra en el estudio de los efectos de la música en el sistema nervioso, las emociones y la personalidad. E-mail: jmupsico1986@gmail.com
} 
seusefeitosnegativos.Entender como você pode desenvolver e melhorar a inteligência emocional também pode ser muito útil para artistas musicais, muitasvezesenfrentamsituaçõesem que eles têm de lidarcommuitasmudançasemocionais e pode chegar a ser dominado por eles. Para entender as emoções e mudanças que podemajudarmaisconhecimentopessoal e para melhorcontrolá-los, permitindo que o músico para se sentir confortávelaojogar e ser capaz de transmitir o que vocêquer que o público.

PALAVRAS CHAVE: Ansiedade. Música. Inteligência Emocional.

\begin{abstract}
This Project focusesonthestudy of performance anxiety and emotionalintelligence in musicians, making a comparisonbetweenclassicalperformers and modernperformers, findingsignificantdifferencesonly in thestateanxietydimension, referred to situations of public performance. Performance anxietyispart of everymusician'slife, to a greaterorlesserextent. Sometimes, ifthereisanexcess of thistype of anxietyit can be a greatproblemforsomemusicians. Thatiswhywebelieveitisreallyimportant to study and delveintotheknowledge of this variable in order to understandbetterits causes and to diminishitsnegativeseffects.Understandinghow to develop and improveemotionalintelligence can be veryhelpful as wellfor musical performers, whohave to faceveryoftensituations in whichtheyhave to dealwithsomeemotionalchangesthat can overwhelmthem. Havingtheability to understandourownemotions and itschangesmay lead to a better personal knowledge and to a better control of them, thatallowsthemusician to feelcomfortablewhileplaying and to be able to communicatewhat he wants to theaudience at thesame time.
\end{abstract}

\title{
KEYWORDS:Anxiety. Music. Emotionalintelligence.
}

\section{INTRODUCCIÓN}

\section{Ansiedad escénica}

El ser humano sufre ansiedad como reacción a una situación que considera amenazante y que piensa que no puede soportar, por lo que tiene miedo a asumirla. Aunque la ansiedad se suela considerar una emoción desagradable, realiza una función muy importante, pues cuando percibimos algo como un peligro, nuestro cuerpo reacciona permaneciendo alerta y preparándose para lo que pueda pasar, por lo que si estuviéramos en peligro de muerte, aumentaríamos nuestras posibilidades de sobrevivir.

Lo que ocurre es que también sentimos ansiedad en otras muchas ocasiones en las que nuestra vida no corre peligro pero reaccionamos como si así fuera y seguimos respondiendo de la misma forma y por lo tanto de manera exagerada e innecesaria ( DALIA,2004,p.30).Es lo que sucede cuando se sufre de ansiedad escénica, entendida como aquella ansiedad que aparece cuando hay que realizar una actividad delante de gente, como hablar en público, dar conciertos, bailar, actuar, realizar deporte, etc.

Este trabajo se centra en la ansiedad escénica de los intérpretes musicales, la cual se produce cuando los músicos identifican la situación de tocar en público como peligrosa. Cuando esto ocurre, el cuerpo reacciona pasando a un estado de alerta, preparándose y poniéndose en tensión, produciéndose una reacción física, cognitiva y emocional que puede llegar a afectar de manera negativa al músico suponiendo un verdadero problema para él, e interfiriendo negativamente en su interpretación. Por ello, es importante el estudio de este 
aspecto, de las diferencias que existen entre los intérpretes, de sus causas y de sus posibles soluciones.

\title{
Inteligencia emocional
}

Fueron Salovey y Mayer los verdaderos creadores e impulsores de éste nuevo término en 1990 con su artículo "EmotionalIntelligence" (ROMERO, 2008, pág. 73), y quienes establecieron una de las definiciones más aceptadas de la inteligencia emocional, refiriéndose a ella como:

\begin{abstract}
La habilidad para percibir, valorar y expresar emociones con exactitud, la habilidad para acceder y/o generar sentimientos que faciliten el pensamiento; la habilidad para comprender emociones y el conocimiento emocional y la habilidad para regular las emociones promoviendo un crecimiento emocional e intelectual (MAYER Y SALOVEY, 1997, apud EXTREMERA \& FERNÁNDEZ BERROCAL, 2004, p. 60-61).
\end{abstract}

Ellos aseguran que el desarrollo de la inteligencia emocional tiene muchas ventajas: aquellas personas con un buen estado de ánimo son más propensas a pensar que los posibles acontecimientos positivos en el futuro son más probables que los negativos, por lo que son capaces de idear planes y así estar mejor preparados;se incrementa el pensamiento creativo, pues cuando se experimenta un estado de ánimo positivo se es capaz de relacionar mejor la información y de buscar soluciones más creativas a los problemas; la atención es dirigida por el estado de ánimo, por lo que cuando se producen emociones fuertes, la atención se dirige a nuevos problemas, y por ello, si se controlan las emociones se puede dirigir la atención a aquello que nos sea más conveniente; se puede conseguir motivarse a través de las emociones y los estados de ánimo, pues una actitud positiva contribuye a una mayor confianza en uno mismo y por tanto a un punto de vista más optimista sobre las situaciones (SALOVEY \& MAYER, 1990, p. 199-200).

En cuanto a la regulación de las emociones de los demás, ambos autores exponen que es una habilidad muy útil y conveniente en muchas situaciones, que pueden ser positivas si quien tiene esa capacidad mejora su estado de ánimo y el de los demás, o negativa si hace lo contrario (SALOVEY \& MAYER, 1990, p. 198)

Esto es muy interesante, sobre todo si lo aplicamos a la capacidad que deben tener los músicos para transmitir contenido emocional al público a través de la música. Si pensamos que existe esa íntima interacción entre las emociones propias y de los demás, y si poseemos la capacidad de influir en ellas, sería de gran utilidad a la hora de interpretar tener la habilidad de conocer nuestros propios sentimientos e identificar los de la gente que nos rodea para así poder modificarlos y crear el ambiente emocional que queremos y estar de esta manera en sintonía con el púbico.

Actualmente están surgiendo estudios relacionados con éstas dos áreas mencionadas. Sobre la ansiedad escénica en músicos, se han realizado estudios considerando y relacionando diferentes variables con la ansiedad escénica, como la relación de ésta con las experiencias negativas vividas (OSBORNE \& KENNY, 2008), con el género (IUSCA \& DAFINOIU, 2011), la relación entre ansiedad estado y ansiedad rasgo (KENNY, DAVIS, \& OATES, 
2004), o la medición de los niveles de ansiedad antes, durante y después de un concierto (PAPAGEORGI, HALLAM, \& WEICH, 2007), entre otros (ZARZA ALZUGARAY, 2012).

Más concretamente, hay algunos estudios que han medido la ansiedad escénica en músicos, intérpretes de flauta travesera, y la frecuencia con la que la experimentan al tocar en público (HERRERA \& MANJÓN, 2013), concluyendo que éstos tenían una mayor preocupación y juicio crítico cuando el concierto era en solitario y ante público en comparación con ensayos o con actuaciones en grupo.

El estudio de la inteligencia emocional está expandiéndose por muchos ámbitos y va adquiriendo relevancia poco a poco. Los ya mencionados Salovey y Mayer sentaron las bases del término con su artículo "EmotionalIntelligence" (ROMERO, 2008, p. 73). Gardner (1993) y su teoría de las inteligencias múltiples incluye a la inteligencia intrapersonal y la inteligencia interpersonal, que se correspondería en cierta medida con lo que llamamos inteligencia emocional, con uno mismo y con los demás. Daniel Goleman (1995), con su libro Inteligencia Emocional, consiguió que gran parte de la población se interesara por este concepto.Boyatzis (1999), con su aplicación de la inteligencia emocional al ámbito laboral, o LeDoux (1996) con su estudio de la fisiología del cerebro y su relación con las emociones, son solo algunos de los autores que han investigado acerca de este concepto en los últimos años (ROMERO, 2008).

Este trabajo pretende evaluar la ansiedad escénica y la inteligencia emocional de intérpretes musicales clásicos y modernos llevando a cabo un trabajo de campo para realizar posteriormente una comparativa entre ambos colectivos y conocer si existen diferencias entre ambos grupos

Se plantean las siguientes hipótesis:

- No existirán diferencias entre intérpretes musicales clásicos y modernos en los niveles de ansiedad entendida como "rasgo".

- Existirán diferencias entre intérpretes clásicos y modernos en los niveles de ansiedad ante situaciones de interpretación musical en público, presentando mayores niveles los intérpretes clásicos.

- Existirán diferencias entre intérpretes clásicos y modernos en los niveles de inteligencia emocional, presentando mayores niveles los intérpretes modernos.

\section{Método}

En el presente trabajo se ha empleado el método correlacional, el cual, se basa en la búsqueda de relaciones entre dos o más variables, analizando en qué medida la variación de unas afectan a otras sin llegar a establecer relaciones causa-efecto (Briones, 1982).

\section{Participantes}

La muestra total consta de 50 participantes, 25 intérpretes de música clásica y 25 intérpretes de música moderna.Del total de la muestra,14eran mujeres (28\%) y 36 hombres 
(72\%), de los cuales 24 (96\%) hombres y 1 (28\%) mujer pertenecían al grupo de intérpretes modernos, y 12 (48\%) hombres y 13 (52\%) mujeres al grupo de intérpretes clásicos.

El rango de edad de los sujetos oscila entre los 18 y los 46 años, con una edad media de 25,3 años, y una desviación típica de 5,96. La media de edad de los intérpretes de música moderna y clásica es de 26,16 años (D.T.=7,12) y 24,44 años (D.T.=4,52), respectivamente.

En el estudio contamos con un mayor número de varones que de mujeres entre los intérpretes modernos debido al escaso número de mujeres matriculadas. Los participantes son en su mayoría españoles, todos estudiantes de la Universidad Alfonso X "El Sabio" de Madrid, de diversos cursos, tanto de Estudios de Grado como de Máster.

\section{Instrumentos}

Para realizar el estudio, hemos utilizado dos pruebas psicométricas, una para cada variable. A continuación, se presentan ambas pruebas, las cuales se han realizado de forma presencial y en papel.

-STAI (Spielberger,2011):

El STAI (State-TraitAnxietyInventory) ${ }^{1}$,diseñado por Spielberg, es un cuestionario que mide dos aspectos diferentes de la ansiedad: ansiedad-rasgo (tendencia general del sujeto a la ansiedad) y ansiedad-estado (ansiedad producida ante situaciones concretas).La prueba está compuesta por 40 ítems con formato de respuesta tipo LIKERT.

-Test TMMS-24:

El TMMS-24(EXTREMERA, FERNÁNDEZ-BERROCAL, \& RAMOS, 2004) ${ }^{2}$ es una prueba de autoinforme que se basa en el "Trait Meta-MoodScale" (TMMS) del grupo de investigación de Salovey y Mayer. Se trata de una escala que evalúa el metaconocimiento de los estados emocionales utilizando 24ítems. Evalúa la inteligencia emocional teniendo en cuenta tres dimensiones, con 8 ítems cada una: atención emocional (capacidad de sentir y expresar los sentimientos de forma adecuada), claridad de sentimientos (capacidad para comprender bien los estados emocionales propios) y regulación o reparación emocional (capacidad para regular los estados emocionales correctamente). Los ítems tienen un formato de respuesta tipo Likert, que va de 1 a 5 puntos, donde los sujetos deben contestar según su grado de acuerdo o desacuerdo con lo expuesto en cada ítem.

\section{Procedimiento}

Para comenzar a realizar el trabajo se escogieron dos tipos de pruebas que evaluaran las variables psicológicas que íbamos a estudiar, la ansiedad (estado y rasgo) y la inteligencia emocional, por lo que se eligieron el STAI y el TMMS-24. Contando en la Facultad de

\footnotetext{
${ }^{1}$ SPIElberger C. D., GORSUCH R. L. y LUSHENE R. E. (2011). STAI, Cuestionario de Ansiedad Estado-Rasgo. Madrid: TEA Ediciones.

${ }^{2}$ EXTREMERA, N., FERNÁNDEZ BERROCAL, P., \& RAMOS, N. (2004). Validity and reliability of the Spanish modified version of the Trait Meta-Mood Scale. PsychologicalReports 94, 751-755.
} 
Música de la Universidad Alfonso $\mathrm{X}$ el Sabio con intérpretes de música clásica y con intérpretes de música moderna, se decidió comparar si los niveles de las variables mencionadas diferían entre ambos grupos.

Para ello se escogieron dos grupos, clásicos y modernos, de 25 sujetos cada uno que participaron voluntariamente, a los que se les pidió que realizaran los test, primero el STAI (ansiedad estado y ansiedad rasgo, en este orden) y luego el TMMS-24. Se les pedía que contestaran a todas las preguntas sin pensar demasiado las respuestas. Antes de comenzar con el apartado de ansiedad estado del test STAI se les pidió a los sujetos que para contestar a las preguntas de esa parte se imaginaran a sí mismos 5 minutos antes de salir al escenario para tocar delante de público en un concierto importante. El estudio se llevó a cabo de forma colectiva en la misma Facultad de Música durante los meses de marzo y abril de 2016.

Posteriormente, se realizó un análisis estadístico de carácter correlacional en el que se compararon entre sí las dos variables estudiadas entre ambos colectivos de músicos.

\section{Análisis estadístico}

Para los análisis estadísticos de los test se ha realizado una comparación de medias mediante la prueba estadística $\mathrm{T}$ de Student.

Debido a que los supuestos que exige la prueba T de Student no se cumplían para dos variables, también pedimos una prueba no paramétrica, llamada U de Mann-Whitney, no encontrándose prácticamente diferencias en comparación con la prueba T de Student.

Para realizar dichos análisis estadísticos se ha utilizado el programa estadístico informático SPSS-PASW (19.0).

\section{Resultados}

En primer lugar se han calculado las escalas generales de los test a partir de la puntuación de los ítems. A continuación, se han obtenido las medias para las escalas generales y para cada uno de los ítems individualmente con el objetivo de hacer una comparativa entre intérpretes clásicos y modernos.

Tras observar los valores de las medias de los dos grupos, el de intérpretes modernos y el de intérpretes clásicos, se comprueba que sólo hay una diferencia de puntuación destacable en la ansiedad estado. En esta escala la media del grupo de músicos modernos es 29,16, y del grupo de músicos clásicos 19,16, estableciéndose una diferencia de 10 puntos.

Tras realizar las anteriormente citadas pruebas estadísticas, se comparan las medias de las variables entre los dos grupos de intérpretes modernos y clásicos, para comprobar si existen diferencias estadísticamente significativas entre ambos grupos.

En la única variable en la que se aprecian diferencias estadísticamente significativas es en ansiedad estado $(\mathrm{p}=0,00)$.

Para la variable atención emocional (ATEM), la media del grupo de moderno es 25,84 y la media del grupo de clásico es 26,28 . El valor de $\mathrm{p}$ es de 0,84 , por lo que podemos concluir que no hay diferencias estadísticamente significativas. 
Para la variable claridad emocional (CLAREM), la media del grupo de moderno es 27,80 y la media del grupo de clásico es 25,12 . El valor de $\mathrm{p}$ es de 0,11 , por lo que podemos decir que no hay diferencias estadísticamente significativas.

Para la variable reparación emocional (REPEM), la media del grupo de moderno es 28,40 y la media de grupo de clásico es 28,16 . El valor de la prueba p es de 0,45 , por lo que vemos que no hay diferencias estadísticamente significativas.

Para la variable ansiedad estado total (AET), la media del grupo de moderno es 19,16 y la media de grupo de clásico es 29,16 . La prueba p es de 0,00 , por lo que vemos que sí hay diferencias estadísticamente significativas.

Para la variable ansiedad rasgo total (ART), la media del grupo de moderno es 19,04 y la media de grupo de clásico es 21,44. El valor de p es de 0,3, por lo que no hay diferencias estadísticamente significativas.

Tabla 1: Media y desviación típica de las variables generales.

\begin{tabular}{|c|c|c|}
\hline Variable & Media & Desviación típica \\
\hline Atención emocional & 26,06 & 7,53 \\
\hline Claridad emocional & 26,46 & 5,95 \\
\hline Reparación emocional & 27,78 & 5,70 \\
\hline Ansiedad estado total & 24,16 & 10,26 \\
\hline Ansiedad rasgo total & 20,24 & 8,54 \\
\hline
\end{tabular}

Fuente: Elaboración propia.

Tabla 2: Media y desviación típica de las escalas generales de los test por grupos.

\begin{tabular}{|c|c|c|c|}
\hline Variable & Grupo & Media & Desviación típica \\
\hline \multirow{2}{*}{ Atención emocional } & Moderno & 25,84 & 7,26 \\
\cline { 2 - 4 } & Clásico & 26,28 & 7,94 \\
\hline \multirow{2}{*}{ Claridad emocional } & Moderno & 27,80 & 4,62 \\
\cline { 2 - 4 } & Clásico & 25,12 & 6,86 \\
\hline \multirow{2}{*}{ Ansiedad estado total } & Moderno & 28,40 & 5,45 \\
\cline { 2 - 4 } & Clásico & 27,16 & 6,00 \\
\cline { 2 - 4 } & Moderno & $\mathbf{1 9 , 1 6}$ & 6,68 \\
\hline \multirow{2}{*}{ Ansiedad rasgo total } & Clásico & $\mathbf{2 9 , 1 6}$ & 10,88 \\
\cline { 2 - 4 } & Moderno & 19,04 & 7,36 \\
\hline
\end{tabular}

Fuente: Elaboración propia.

Tabla 3: Valores de la prueba T de Student (t) y de la significación bilateral (p).

\begin{tabular}{|c|c|c|}
\hline Variable & $\mathbf{t}$ & $\mathbf{p}$ \\
\hline Atención emocional & $-0,20$ & 0,84 \\
\hline Claridad emocional & 1,62 & 0,11 \\
\hline Reparación emocional & 0,77 & 0,45 \\
\hline Ansiedad estado total & $-3,92$ & 0,00 \\
\hline Ansiedad rasgo total & $-0,99$ & 0,33 \\
\hline
\end{tabular}

Fuente: Elaboración propia. 
A parte de las escalas generales, se han comparado los ítems individualmente, para lo cual hemos contrastado las mediasde cada ítempara los grupos de clásico y de moderno y observamos en cuales la diferencia es mayor. Hemos considerado que la diferencia entre las medias es significativa cuandosu valor está por encima de 0,50 .

Los ítems que presentan una diferenciaestadísticamente significativa entre sus medias son:AE5 ("me siento cómodo, estoy a gusto"), AE7 ("estoy preocupado ahora por posibles desgracias futuras"), AE9 ("me siento angustiado"), AE10 ("me siento confortable"), AE11 ("tengo confianza en mí mismo"), AE15 ("estoy relajado"), AE16 ("me siento satisfecho"), AE19 ("me siento alegre"), AE20 ("en este momento me siento bien"), AR31 ("suelo tomar las cosas demasiado seriamente"), AR40 ("cuando pienso sobre asuntos y preocupaciones actuales, me pongo tenso y agitado"), TMMS8 ("presto mucha atención a cómo me siento"), TMMS16 ("puedo llegar a comprender mis sentimientos"), TMMS17 ("aunque a veces me siento triste, suelo tener una visión optimista" y TMMS22 ("me preocupo por tener un buen estado de ánimo").

La mayor parte de los ítems en los que se presentan diferencias notorias entre las medias de cada grupo pertenecen al STAI, en concreto a la escala de ansiedad estado, aunque también hay diferencias en algunos ítems de las escalas de ansiedad rasgo del STAI y en algunos del TMMS-24.

Tabla 4: Medias de las respuestas de cada uno de los ítems en los grupos clásico y moderno, y diferencia entre ambas medias

\begin{tabular}{|c|c|c|c|}
\hline Ítem & Media grupo Moderno & Media grupo Clásico & $\begin{array}{c}\text { Diferencia entre } \\
\text { medias }\end{array}$ \\
\hline AE1 & 1,44 & 1,04 & 0,40 \\
\hline AE2 & 1,88 & 1,48 & 0,40 \\
\hline AE3 & 1,12 & 1,44 & 0,32 \\
\hline AE4 & 0,40 & 0,80 & 0,40 \\
\hline AE5 & 2,16 & 1,16 & 1,00 \\
\hline AE6 & 1,04 & 1,20 & 0,16 \\
\hline AE7 & 0,60 & 1,40 & 0,80 \\
\hline AE8 & 1,57 & 1,32 & 0,25 \\
\hline AE10 & 0,36 & 0,92 & 0,56 \\
\hline AE11 & 1,72 & 1,00 & 0,72 \\
\hline AE12 & 2,12 & 1,44 & 0,68 \\
\hline AE13 & 1,20 & 1,44 & 0,24 \\
\hline AE14 & 0,72 & 0,84 & 0,12 \\
\hline AE15 & 0,40 & 0,68 & 0,28 \\
\hline AE16 & 1,52 & 0,92 & 0,60 \\
\hline AE17 & 1,60 & 1,08 & 0,52 \\
\hline AE19 & 1,00 & 1,36 & 0,36 \\
\hline AE20 & 0,80 & 1,00 & 0,20 \\
\hline AR21 & 2,20 & 1,24 & 0,96 \\
\hline AR22 & 2,28 & 1,24 & 1,04 \\
\hline AR23 & 2,36 & 2,28 & 0,08 \\
\hline AR24 & 0,88 & 0,88 & 0,00 \\
\hline & 0,32 & 0,48 & 0,16 \\
\hline & 0,80 & 0,48 & 0,32 \\
\hline
\end{tabular}




\begin{tabular}{|c|c|c|c|}
\hline AR25 & 1,00 & 0,92 & 0,08 \\
\hline AR26 & 1,56 & 1,48 & 0,08 \\
\hline AR27 & 2,08 & 1,72 & 0,36 \\
\hline AR28 & 0,84 & 1,04 & 0,20 \\
\hline AR29 & 1,00 & 1,32 & 0,32 \\
\hline AR30 & 2,48 & 2,28 & 0,20 \\
\hline AR31 & 1,32 & 1,92 & 0,60 \\
\hline AR32 & 1,28 & 1,40 & 0,12 \\
\hline AR33 & 2,04 & 1,80 & 0,24 \\
\hline AR34 & 0,80 & 0,84 & 0,04 \\
\hline AR35 & 0,88 & 0,80 & 0,08 \\
\hline AR36 & 1,88 & 1,84 & 0,04 \\
\hline AR37 & 1,24 & 1,40 & 0,16 \\
\hline AR38 & 1,00 & 0,72 & 0,28 \\
\hline AR39 & 2,00 & 2,08 & 0,08 \\
\hline AR40 & 1,16 & 1,84 & 0,68 \\
\hline TMMS1 & 3,80 & 3,56 & 0,24 \\
\hline TMMS2 & 3,52 & 3,36 & 0,16 \\
\hline TMMS3 & 3,48 & 3,16 & 0,32 \\
\hline TMMS4 & 3,64 & 3,72 & 0,08 \\
\hline TMMS5 & 3,04 & 2,96 & 0,08 \\
\hline TMMS6 & 2,40 & 2,84 & 0,44 \\
\hline TMMS7 & 3,16 & 3,36 & 0,20 \\
\hline TMMS8 & 2,80 & 3,32 & 0,52 \\
\hline TMMS9 & 3,32 & 3,24 & 0,08 \\
\hline TMMS10 & 3,44 & 3,20 & 0,24 \\
\hline TMMS11 & 3,64 & 3,28 & 0,36 \\
\hline TMMS12 & 3,56 & 3,24 & 0,32 \\
\hline TMMS13 & 3,56 & 3,20 & 0,36 \\
\hline TMMS14 & 3,20 & 2,76 & 0,44 \\
\hline TMMS15 & 3,16 & 2,92 & 0,24 \\
\hline TMMS16 & 3,92 & 3,28 & 0,64 \\
\hline TMMS17 & 3,84 & 3,28 & 0,56 \\
\hline TMMS18 & 3,36 & 3,44 & 0,08 \\
\hline TMMS19 & 2,76 & 2,76 & 0,00 \\
\hline TMMS20 & 3,48 & 3,40 & 0,08 \\
\hline TMMS21 & 3,36 & 3,24 & 0,12 \\
\hline TMMS22 & 3,72 & 3,16 & 0,56 \\
\hline TMMS23 & 4,64 & 4,32 & 0,32 \\
\hline TMMS24 & 3,24 & 3,56 & 0,32 \\
\hline
\end{tabular}

Fuente: Elaboración propia.

Aparecen subrayados los ítems en los que se observan mayores diferencias entre la media de ambos grupos. 


\section{Discusión}

Después de haber realizado el experimento podríamos decir que los resultados que hemos obtenido confirman parcialmente las hipótesis planteadas inicialmente. A continuación,se comentan los resultados y se explican si se cumplen o no las hipótesis y en qué medida.

Hipótesis 1: No existirán diferencias entre intérpretes musicales clásicos y modernos en los niveles de ansiedad entendida como "rasgo".

Se cumple parcialmente esta hipótesis, ya que no existen diferencias estadísticamente significativas en la escala de ansiedad-rasgo entre ambos grupos. Atendiendo a los ítems que pertenecen a esta escala de forma individual, podemos decir que en general no se encuentran apenas diferencias entre los intérpretes clásicos y modernos en cuanto a ansiedad rasgo, excepto en dos de ellos. Uno de esos ítems es

Uno de esos ítems es el AR31 ("suelo tomar las cosas demasiado seriamente"), en el que existe una diferencia de 0,60 puntos entre las medias de los dos grupos, donde los de clásico obtienen mayor puntuación $(1,92)$ que los de moderno $(1,32)$. Esto podría deberse a que los intérpretes de música clásica suelen estar inmersos en un ambiente más formal, en el que se forman para actuar en conciertos delante de un público determinado que a veces puede incluir a sus propios profesores, donde suele primar la correcta ejecución y donde hay un cierto "protocolo" que puede crear una sensación de seriedad. Por el contrario,en los conciertos de música moderna se crea un ambiente más distendido en el que el público se involucra más activamente y el intérprete goza de más libertad y puede sentirse quizás más cómodo y arropado. Incluso socialmente, la música clásica se suele asociar a contextos más formales y la música moderna a ambientes más informales.

Hipótesis 2:Existirán diferencias entre intérpretes clásicos y modernos en los niveles de ansiedad ante situaciones de interpretación musical en público, presentando mayores niveles los intérpretes clásicos.

La hipótesis se cumple en su totalidad ya que existen diferencias estadísticamente significativas considerando la escala general de ansiedad-estado. Los ítems de esta escala en los que se encuentran las diferencias más acusadas entre ambos grupos son: AE5, AE7, AE9, AE10, AE11, AE15, AE16, AE19 y AE20.

El ítem en el que la diferencia entre los intérpretes clásicos y modernos ha sido más acusada ha sido en el AE20 ("en este momento me siento bien"), con un valor de 1,04, en el que los intérpretes modernos han obtenido una media más alta $(2,28)$, casi el doble, que los clásicos $(1,24)$. Por lo tanto, se concluye que los intérpretes modernos se sienten bien antes de una actuación ante el público, mucho mejor que los intérpretes clásicos.

Estos resultados podrían deberse a varios factores. Uno de ellos podría ser la actitud antes de salir al escenario, pues como ya dijimos anteriormente, los músicos clásicos suelen estar preocupados ya que suelen tocar en contextos más serios, donde habitualmente van a ser evaluados, por lo que tienen miedo de cometer fallos, de decepcionar a sus profesores o de las posibles malas críticas que pueden recibir después.

Sin embargo, pensamos que los intérpretes modernos mantienen otra actitud antes de actuar, más abierta a la experiencia, a disfrutar de ese momento y a involucrar al público y hacerlo partícipe de la música, sin estar tan preocupados por ser juzgados como los clásicos y 
teniendo la capacidad de aprovechar la ocasión para crecerse como músicos. Así lo pensabanSalovey y Mayer:

Por ejemplo, algunos individuos pueden canalizar la ansiedad creada por las situaciones de evaluación tales como ensayos y actuaciones inminentes para motivarles a preparar más a fondo y alcanzar los estándares más exigentes...Las personas pueden usar buenos estados de ánimo para aumentar la confianza en sus capacidades y así persistir en el enfrentamiento de los obstáculos y las experiencias aversivas (1990, p. 200).

También es cierto que los músicos modernos suelen tocar, además de en entornos académicos, en otros ambientes más ociosos, y así tienden a asociar la situación de tocar ante el público con sentimientos y experiencias positivas, disminuyendo de esta manera la ansiedad al exponerse con más frecuencia al público y "acostumbrándose" así a esa situación.

Hipótesis 3:Existirán diferencias entre intérpretes clásicos y modernos en los niveles de inteligencia emocional, presentando mayores niveles los intérpretes modernos.

Podemos decir que la hipótesis no se cumple ya que no existen diferencias estadísticamente significativas entre ambos grupos en ninguna de las tres escalas generales de la prueba.Además, solo se aprecian diferencias entre ambos grupos en cuatro ítems de la prueba y sólo en tres de los cuatro ítems los intérpretes modernos presentan mayores niveles de inteligencia emocional.

Los ítems en los que se han encontrado diferencias son: TMMS8, TMMS16, TMMS17, TMMS22.

\section{CONCLUSIONES}

Podemos decir que los resultados de nuestro estudio son coherentes a nivel general con las hipótesis planteadas al inicio, y consideramos que se han cumplido de manera satisfactoria los objetivos principales de este trabajo.

Hemos podido conocer en profundidad y ser conscientes de la importancia de la inteligencia emocional y de la ansiedad escénica en la vida de un intérprete musical, y de cómo afectan en situaciones como los conciertos ante el público, donde los músicos se sienten expuestos y donde estos dos factores son determinantes para poder afrontarlas. La ansiedad escénica se puede convertir en un problema si no se conocen las estrategias necesarias para controlar nuestro cuerpo, nuestros pensamientos y nuestras emociones. Aquí entra en juego el desarrollo de la inteligencia emocional para poder percibir y ser conscientes de nuestros estados emocionales para así ser capaces de regularlos y modificarlos cuando sea necesario.

Algunas de las causas que podrían determinar los resultados obtenidos podrían ser: la actitud con la que el intérprete se enfrenta a una actuación en público, la frecuencia con la que se expone ante el público, los contextos en los que suele tocar, las experiencias que ha tenido interpretando delante de gente, la dependencia que tiene de la partitura y la capacidad de improvisación, si toca en un grupo o lo hace como solista, la implicación del público en el concierto, la capacidad para conocer sus propias emociones y las del público y tener la habilidad de regularlas. 
Consideramos, por tanto, necesario tomar medidas para desarrollar la inteligencia emocional y controlar la ansiedad escénica, y aquí proponemos algunas de ellas.

Pensamos que es necesario potenciar la relevancia de la inteligencia emocional y trabajar en su desarrollo desde los primeros años de estudio, pues es de gran utilidad en el campo musical pero también en cualquier otro ámbito, ya que nos facilita el propio conocimiento de nuestros estados emocionales y entender cómo nos sentimos en cada momento. Pero también nos ayuda a relacionarnos socialmente, pudiendo percibir mejor los sentimientos de los demás. Esta capacidad nos posibilita modificar las emociones propias y las de los demás, y de esta manera adaptarnos adecuadamente a las diversas situaciones en las que nos encontramos diariamente. Es por tanto un factor determinante en la vida de un intérprete, que se expone continuamente a la opinión y al juicio de los demás, y si se trabaja y se potencia adecuadamente se puede obtener una mayor capacidad de adaptación a las situaciones a las que deberá enfrentarse el estudiante de música en esta profesión.

Ocurre algo parecido con la ansiedad escénica, pues es un aspecto que está emergiendo actualmente en los conservatorios de música y al que se le debe prestar mucha atención pues es uno de los principales problemas de la disminución del rendimiento en las actuaciones con público. Hay grandes músicos y buenos estudiantes que trabajan intensamente para conseguir una buena interpretación y que luego ven truncado ese objetivo debido a la ansiedad escénica que sufren. Si desde los primeros años de estudio musical se dedica un tiempo a explicar en qué consiste la ansiedad escénica y cómo se puede controlar o mermar sus efectos negativos, se avanzará mucho evitando futuros problemas de este tipo que derivarían en una mejor interpretación y mayor seguridad del músico ante el público, consiguiendo además un mejor control ante situaciones similares, como hablar en público, que se presentarán a lo largo de los años.

Otra mejora importante podría darse en cuanto a la frecuencia en la exposición ante el público, que consideramos insuficiente en la mayoría de entornos académicos. La escasez de oportunidades que se les da a los alumnos para tocar en público favorece la aparición de la ansiedad escénica, pues ellos no ven esta situación como habitual y por lo tanto no se llegan a acostumbrar a ella y a las sensaciones que les produce.

Es importante que se impulsen iniciativas para que los estudiantes puedan tocar en un escenario cada poco tiempo, y que lo consideren una oportunidad para mostrar su trabajo a los demás y así aprender y mejorar, y no sentirse juzgados o inseguros cada vez que esto ocurra. Si además se dedicara un espacio en el currículo escolar para aprender a controlar la ansiedad escénica y a desarrollar las capacidades de inteligencia emocional, se producirían grandes avances y mejoras en cuanto a la interpretación en público. Se podría conseguir, por ejemplo, explicando las causas de la ansiedad, entendiendo cómo y qué la produce y qué se puede hacer para controlar sus efectos, conociendo nuestras propias emociones y sentimientos y las de los demás, aprendiendo a regularlos y que de esta manera no nos desborden en situaciones difíciles, mejorando nuestra relación con los demás identificando sus estados emocionales y aplicando todo esto a la interpretación musical.

\section{REFERÊNCIAS}


BOYATZIS, R. E. Self-directed change and learnings as a necesary meta-competency for success and effectiveness in the 21st century. En R. Sims, \& J. Veres, Keys to employee success in the coming decades,págs. 15-32, Westport: Greedwood.1999.

BRIONES, G. Métodos y técnicas de investigación para las ciencias sociales. México: Trillas. 1982.

CANO, A.. Sociedad Española para el Estudio de la Ansiedad y el Estrés. Obtenido de http://www.ansiedadyestres.org/content/que_es_la_ansiedad 8 de agosto de 2016.

DALIA, G. Cómo superar la ansiedad escénica en músicos. Madrid: Mundimúsica Ediciones.2004.

DALIA, G., \& Pozo, A. El músico. Una introducción a la psicología de la interpretación musical. Madrid: Mundimúsica Ediciones.2006.

EXTREMERA, N., \& FERNÁNDEZ BERROCAL, P. El uso de las medidas de habilidad en el ámbito de la inteligencia emocional. Ventajas e inconvenientes con respecto a las medidas de auto-informe. Boletín de Psicología, 80, 59-77, marzo de 2004.

EXTREMERA, N., FERNÁNDEZ-BERROCAL, P., \& RAMOS, N. Validity and reliability of the Spanish modified version of the Trait Meta-Mood Scale. Psychological Reports 94, 751-755.2004.

GARCÍA, M. C. Inteligencia Emocional: Estudiando otras perspectivas. UMBRAL. Revista de Educación, Cultura y Sociedad, 3(4), 143-148, marzo de 2003.

GARDNER, H. Inteligencias múltiples: la teoría en la práctica. Barcelona: Paidós.1993.

GOLEMAN, D.Inteligencia Emocional. Barcelona: Kairós.1995.

HERRERA, L., \& MANJÓN, G. J. Ansiedad escénica musical en estudiantes de flauta travesera. Eufonía. Didáctica de la música 57, 43-55.enero de 2013. 
IBARROLA, B.. ENTREVISTA Begoña Ibarrola, psicóloga e investigadora. (N. G. Uriarte, Entrevistador) Obtenido de http://www.eldiario.es/norte/euskadi/sistema-educativo-incorporeinteligencia-emocional_0_486101594.html, 21 de febrero de 2016.

IUSCA, D., \& DAFINOIU, I. Performance anxiety and musical level of undergraduate students in exam situations: the role of gender and musical instrument. Procedia - Social and Behavioral Sciences, 33 , $448-455,2011$.

KENNY, D. T., DAVIS, P., \& OATES, J. Music performance anxiety and occupational stress amongst opera chorus artists and their relationship with state and trait anxiety and perfectionism. Journal of Anxiety Disorders, 18, 757-777.2004.

LEDOUX, J. E. El cerebro emocional.Barcelona: Ariel.1996.

NAGY, J. El miedo escénico. Temas para la Educación(26), 1-5. Obtenido de http://www2.fe.ccoo.es/andalucia/docu/p5sd10781.pdf, enero de 2014.

OSBORNE, M., \& KENNY, D. T. The Role of Sensitizing Experiences in Music Performance Anxiety in Adolescent Musicians. Psychology of Music, 36(4), 447-462,2008.

PAPAGEORGI, I., HALLAM, S., \& WEICH, G. A Conceptual Framework for Understanding Musical Performance Anxiety. Research Studies in Music Education, 28, 83107., 2007.

POZO, A. Técnicas cognitivas para superar la ansiedad escénica. Obtenido de http://conservatoriosegovia.centros.educa.jcyl.es/sitio/upload/CAPITULO_6_Tecnicas_cogn itivas.pdf, s.f

ROBLES, J., \& PORTELLANO, J. A. Influencia de la experiencia musical sobre las funciones ejecutivas y el nivel de ansiedad. (Tesis Doctoral). Universidad Complutense de Madrid, Madrid: Polibea.2012.

ROJAS, E. La ansiedad. Madrid: Ediciones Temas de Hoy.2009.

ROMERO, M. A. La Inteligencia Emocional: abordaje teórico. Anuario de Psicología Clínica $y$ de la salud, 4, 73-76, 2008. 
SALOVEY, P., \& MAYER, J. D. Emotional Intelligence. 185-211.1990.

SPIELBERGUER, C. D., GORSUCH, R. L., \& LUSHENE, R. E. STAI, Cuestionario de Ansiedad Estado-Rasgo. Madrid: TEA Ediciones.2011.

ZARZA ALZUGARAY, F. J. La ansiedad escénica en músicos de Grado Superior y su relación con el optimismo disposicional. (Trabajo Fin de Máster). Universidad de Zaragoza, Zaragoza.2012.

Recebido em: 12 de março/2018

Aprovando em: 08 de junho/2018 